\title{
Open Clusters as tracers of the Galactic disk: the Bologna Open Clusters Chemical Evolution project
}

\author{
Angela Bragaglia ${ }^{1}$, Eugenio Carretta ${ }^{1}$, Raffaele Gratton ${ }^{2}$ \\ and Monica Tosi ${ }^{1}$ \\ ${ }^{1}$ INAF-Osservatorio Astronomico di Bologna \\ via Ranzani 1, I-40127 Bologna, Italy \\ email: angela.bragaglia, eugenio.carretta, monica.tosi @oabo.inaf.it \\ ${ }^{2}$ INAF-Osservatorio Astronomico di Padova \\ vicolo dell'Osservatorio 5, I-35122 Padova, Italy \\ email: raffaele.gratton@oapd.inaf.it
}

\begin{abstract}
We present an update of the Bologna Open Clusters Chemical Evolution project (BOCCE, in short). We are conducting a photometric and spectroscopic survey of Open Clusters, to be used as tracers of the Galactic disk properties and evolution. We obtain the clusters parameters (age, distance, metallicity, and detailed abundances) in a precise and homogeneous way. We have collected data for about 40 Open Clusters and have fully analyzed the photometric data for about one half and the spectra for one quarter of them. We present here results based on these works and indicate what will come next.
\end{abstract}

Keywords. techniques: photometric, techniques: spectroscopic, stars: abundances, HertzsprungRussell diagram, Galaxy: abundances, Galaxy: disk, open clusters and associations: general

\section{Overview}

Open Clusters (OCs) are very useful tracers of the properties of the Galactic disk (e.g., Friel 1995), because they are seen over the entire disk, their ages and distances are generally measurable with better precision than those of isolated field stars up to large distances, and they cover the entire range of metallicities and ages of the disk.

In particular, OCs are very important to describe the metallicity (and detailed abundances) distribution of the disk, a key ingredient for models of chemical evolution. The metallicity distribution can be traced by other objects, like young stars, Cepheids, and Planetary Nebulae, but they are all rather young and can be used to describe the presentday situation, or the one in the near past. Instead, OCs go back to the first epochs of the disk, so we have access to the history of the disk formation and evolution and we may follow whether and how the metallicity distribution changed with time.

In the past, OCs have been used to define the metallicity distribution, with controversial results. The most commonly held view is that of a negative radial gradient (e.g., Friel et al. 2002), but an alternative picture, with two flat distributions at about solar and sub-solar metallicity and a discontinuity near a $R_{\mathrm{GC}}$ of $10 \mathrm{kpc}$, has been presented (Twarog, Ashman \& Anthony-Twarog 1997). More recently, the observation of far-away OCs has permitted to reach $\mathrm{R}_{\mathrm{GC}}>20 \mathrm{kpc}$; these studies seem to indicate a negative gradient in the inner region and a flattening in the outer disk, with the transition at 10-14 kpc, see Yong, Carney \& Teixera de Almeida (2005), Carraro et al. (2007), Sestito et al. (2008). 
Table 1. OCs for which photometry and/or spectroscopy has been obtained. In all cases where the analysis has already been completed we give the references. Photometric parameters shown here refer to the (old) Padova tracks to give a ranking. More OCs are in our sample but the data quality has not been checked yet. In three cases (Be 19, NGC 6603, and Cr 110) telescope time has been granted but spectroscopic observations have not been carried out yet. Results obtained within a parallel collaboration (see Randich et al. 2005) are indicated within square brackets, since they are not strictly on the BOCCE spectroscopic scale; Cr 261, NGC 3960, NGC 6253 were observed in both programs.

\begin{tabular}{|c|c|c|c|c|c|c|c|c|}
\hline Cluster & photom. & publ.? & $(\mathrm{m}-\mathrm{M})_{0}$ & $\mathrm{E}(\mathrm{B}-\mathrm{V})$ & Z & $\begin{array}{l}\text { age } \\
\text { (Gyr) }\end{array}$ & high-res spec. & {$[\mathrm{Fe} / \mathrm{H}]$} \\
\hline NGC 752 & - & - & & & & & SARG@TNG & - \\
\hline Be 66 & TNG & (1) & 13.20 & 1.22 & 0.008 & 3.80 & no & - \\
\hline NGC 1193 & TNG & no & & & & & no & - \\
\hline Be 17 & TNG & $(2)$ & 12.20 & 0.62 & 0.008 & 8.50 & SARG@TNG & - \\
\hline Be 19 & TNG & in prep & & & & & SARG@TNG? & - \\
\hline Be 20 & TNG & (1) & 14.70 & 0.13 & 0.008 & 5.80 & [FLAMES] & {$[-0.30(6)]$} \\
\hline Be 21 & Danish & (3) & 13.50 & 0.78 & 0.004 & 2.20 & no & - \\
\hline NGC 2099 & CFHT & $(3)$ & 10.50 & 0.36 & 0.008 & 0.43 & SARG@TNG & - \\
\hline Be 22 & NTT & (3) & 13.80 & 0.64 & 0.020 & 2.40 & no & - \\
\hline NGC 2168 & CFHT & (3) & 9.80 & 0.20 & 0.008 & 0.18 & SARG@TNG & - \\
\hline NGC 2204 & WFI & no & & & & & UVES & in prep \\
\hline NGC 2243 & Danish & (3) & 12.74 & 0.08 & 0.004 & 4.80 & FLAMES/SV & - \\
\hline $\operatorname{Tr} 5$ & WFI & no & & & & & (archive) & - \\
\hline Cr 110 & DFOSC & (3) & 11.45 & 0.57 & 0.004 & 1.70 & SARG@TNG? & - \\
\hline NGC 2266 & TNG & no & & & & & SARG@TNG & - \\
\hline Biu $11 /$ Be 27 & SuSI2 & no & & & & & no & - \\
\hline Be 29 & NTT & (3) & 15.60 & 0.12 & 0.004 & 3.70 & [FLAMES] & {$[-0.31(6)]$} \\
\hline Be 32 & $\mathrm{TNG}$ & (4) & 12.60 & 0.12 & 0.008 & 5.20 & [FLAMES] & {$[-0.29(7)]$} \\
\hline Biu 13/Be 34 & SuSI2 & no & & & & & no & - \\
\hline NGC 2323 & CFHT & (3) & 10.20 & 0.22 & 0.020 & 0.12 & no & - \\
\hline To 2 & Danish & in prep & & & & & [FLAMES] & - \\
\hline NGC 2324 & WFI & no & & & & & [FLAMES] & {$[-0.17(8)]$} \\
\hline Вe 36 & SuSI2 & no & & & & & no & - \\
\hline Mel 66 & - & - & & & & & [FLAMES] & {$[-0.33(6)]$} \\
\hline Mel 71 & Danish & in prep & & & & & FEROS & in prep \\
\hline NGC 2477 & - & - & & & & & [FLAMES] & {$[+0.07(8)]$} \\
\hline NGC 2506 & mosaic & (3) & 12.60 & 0.00 & 0.020 & 1.70 & FEROS & $-0.20(9)$ \\
\hline Pi 2 & Danish & (3) & 12.70 & 1.29 & 0.020 & 1.10 & no & - \\
\hline NGC 2660 & Dutch & (3) & 12.30 & 0.40 & 0.020 & 0.95 & [FLAMES] & {$[+0.04(7)]$} \\
\hline NGC 2849 & Dutch & no & & & & & no & - \\
\hline NGC 3960 & DFOSC & (5) & 11.60 & 0.29 & 0.020 & 0.90 & FEROS & $-0.12(5)$ \\
\hline Cr 261 & mosaic & (3) & 12.20 & 0.30 & 0.020 & 6.00 & FEROS & $-0.03(10)$ \\
\hline NGC 4815 & FORS & no & & & & & no & - \\
\hline NGC 6134 & DFOSC & no & & & & & FEROS & $+0.15(9)$ \\
\hline NGC 6253 & Danish & (3) & 11.00 & 0.23 & 0.050 & 3.00 & FEROS+UVES & $+0.46(11)$ \\
\hline IC 4651 & DFOSC & no & & & & & FEROS & $+0.11(9)$ \\
\hline NGC 6603 & Danish & no & & & & & SARG@TNG? & - \\
\hline IC 4756 & DFOSC & no & & & & & FEROS & - \\
\hline NGC 6791 & CFHT & in prep & & & & & SARG@TNG & $+0.47(12)$ \\
\hline IC 1311 & TNG & no & & & & & no & - \\
\hline NGC 6819 & CFHT & $(3)$ & 12.20 & 0.12 & 0.020 & 2.00 & SARG@TNG & $+0.07(13)$ \\
\hline NGC 6939 & TNG & (3) & 11.30 & 0.34 & 0.020 & 1.30 & SARG@TNG & - \\
\hline King 11 & TNG & (4) & 11.75 & 1.04 & 0.010 & 4.25 & no & - \\
\hline NGC 7789 & CFHT & no & & & & & SARG@TNG & - \\
\hline NGC 7790 & Loiano & (3) & 12.65 & 0.54 & 0.020 & 0.10 & SARG@TNG & - \\
\hline
\end{tabular}

(1) Andreuzzi, Bragaglia \& Tosi (2008); (2) Bragaglia et al. (2006b); (3) Bragaglia \& Tosi (2006), where references to the original papers are given; (4) Tosi et al. (2007); (5) Bragaglia et al. (2006a); (6) Sestito et al. (2008); (7) Sestito et al. (2006); (8) Bragaglia et al. (2008); (9) Carretta et al. (2004); (10) Carretta et al. (2005); (11) Carretta, Bragaglia \& Gratton (2007);

(12) Gratton et al. (2006); (13) Bragaglia et al. (2001) 


\section{The BOCCE survey}

We have started a survey of OCs to derive in the most precise and homogeneous way their main parameters: age, distance, reddening, metallicity (and detailed abundances). As one of our main interest is the chemical evolution of the disk, we named our survey the "Bologna Open Clusters Chemical Evolution" project, BOCCE in short. Our goal is to build a sample large enough to be representative of the whole cluster population (in age, metallicity and position in the Galaxy). Of course, if we want to study the history of the disk we have to concentrate on old OCs: of the about 120 OCs with age larger than 1 Gyr found in the Dias et al. (2002) catalogue, we have collected data for a fair fraction, and have already studied 16 clusters in detail. We employ:

- Photometry and the Synthetic Colour-Magnitude diagrams technique to derive at the same time age, distance, reddening and a first indication of metallicity; for a review of the method and results see Bragaglia \& Tosi (2006). This is the more advanced part of our survey, having already published results for more than half the original sample.

- Medium resolution spectroscopy to derive radial velocities, hence membership, for stars in crucial evolutionary phases, like the main sequence Turn-Off or the Red Giant Branch; for an application, see e.g. D'Orazi et al. (2006). This is a secondary part of our project.

- High resolution spectroscopy to measure chemical abundances, using both equivalent widths and spectrum synthesis; for a presentation of the method, see Bragaglia et al. (2001), Carretta et al. (2004). This part of the work started later and we still have to catch up with the photometric survey, but we have recently acquired spectra for many OCs. We also plan to use archive data and homogenize results obtained within a parallel program (see Randich et al. 2005).

Table 1 shows the present status of our project; in summary we have observed clusters with ages from 0.1 to $9 \mathrm{Gyr}, \mathrm{R}_{\mathrm{GC}}$ from about 7 to $21 \mathrm{kpc}$, and metallicity from less than half solar to more than double solar.

We have a few very interesting objects in our sample, e.g., Be 17 and NGC 6791 (the oldest OCs known, with age near $9 \mathrm{Gyr}$ ) or Be 20, Be 29 (with $\mathrm{R}_{\mathrm{GC}}$ of abour 16 and $21 \mathrm{kpc}$ ) and $\mathrm{Cr} 261$, NGC 6253 (inside the solar circle) very important to define the metallicity gradient. Two of these clusters, NGC 6791 and NGC 6253, are also the most metal-rich OC presently known.

In the Table, to give an uniform ranking of properties, we put the values derived from photometry using the (old) Padova tracks (e.g., Bressan et al. 1993), but we always use three sets of evolutionary tracks (without overshooting and with two treatment of it). This way we are better able to quantify the systematics between results based on different stellar models. We always use the same sets of tracks even if a newer version of the same code has become available (as in the case of the Padova models) to maintain the maximum homogeneity in our determinations $\dagger$

The metallicity shown in the last column of Table 1 has been obtained from the high resolution spectra. We observe a few stars per clusters, chosen if possible among confirmed members; we usually target Red Clump stars since they are the best compromise between luminosity and temperature: we can obtain high $\mathrm{S} / \mathrm{N}$ spectra of stars not too cool to be a problem for model atmospheres. Figure 1 (left panel) shows an example of the quality of our data in the case of the two very metal-rich clusters NGC 6253 and NGC 6791 . We

$\dagger$ An exception will be NGC 6791, for which we are employing new tracks at $Z=0.04$ or 0.05 , computed on purpose or retrieved from recent publications, because of the combination of very high metallicity and old age. 

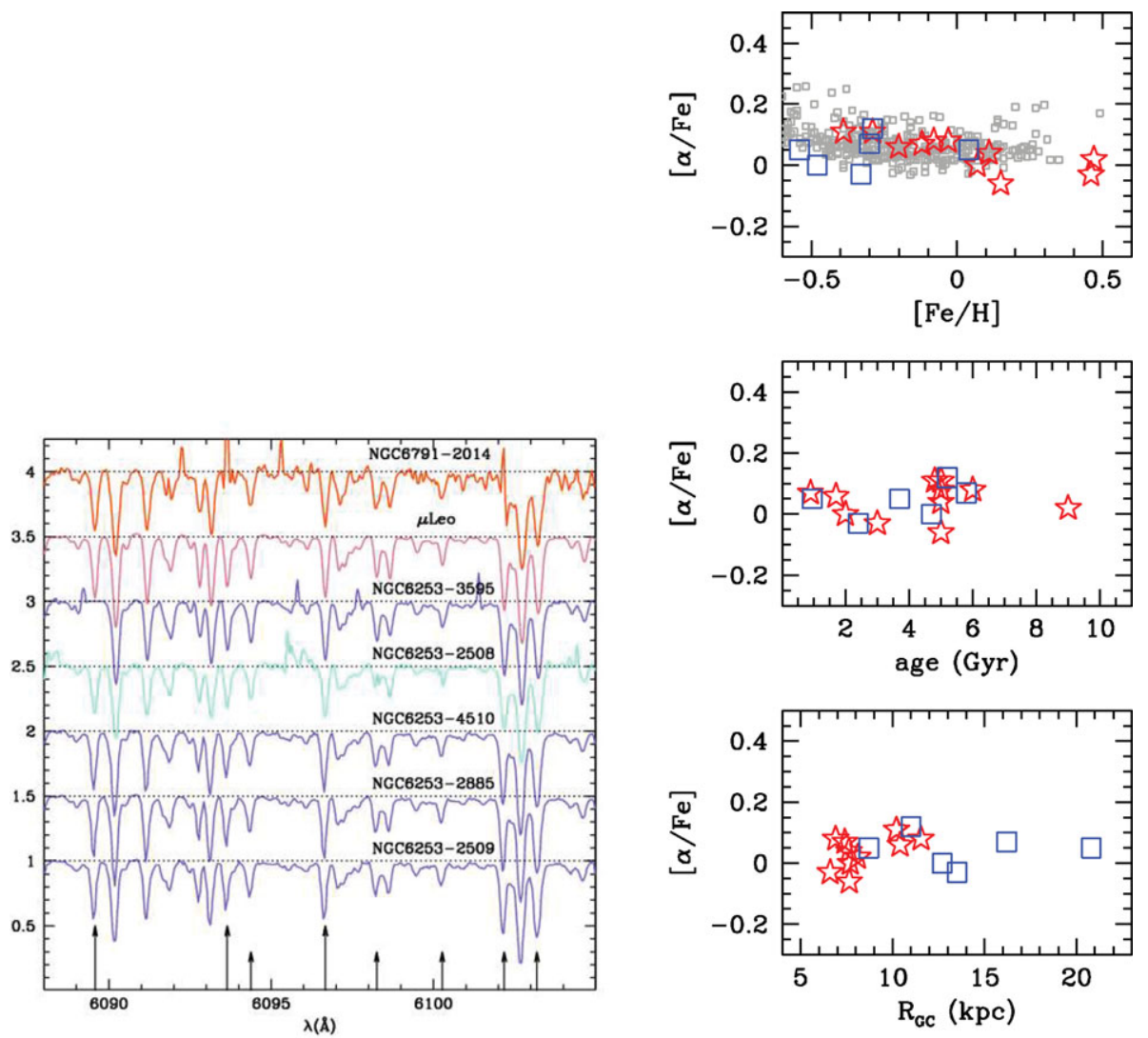

Figure 1. Left panel: Spectra obtained with FEROS and UVES for NGC 6253 (Carretta et al. 2007), with SARG for NGC 6791 (Gratton et al. 2006); also shown is the very metal-rich field giant $\mu$ Leo. The arrows indicate lines used in the abundance determination. Right panels: $[\alpha / \mathrm{Fe}]$ ratio for OCs in the BOCCE sample, compared to thin disk field stars (upper plot) and shown in fuction of age (middle plot) and $\mathrm{R}_{\mathrm{GC}}$ (lower plot). Red stars indicate abundances strictly on the BOCCE scale, open squares are from Sestito et al. (2006), Sestito et al. (2008), Bragaglia et al. (2008) for four OCs (from the parallel program), and Villanova et al. (2005), Carraro et al. (2004) for two OCs.

try to maintain also in this case the most homogeneous procedure, using the same line lists, $g f$ 's, model atmospheres, solar reference values, way to measure equivalent widths, synthesis, and kind of stars.

Since the photometric and spectroscopic parts of our programs are not "aligned" yet, we are not presently able to derive in a fully self-consistent way the metallicity gradient or the properties of our whole sample. However, putting together our results, literature ones, and the FLAMES survey, we confirm the presence of a radial metallicity gradient flattening in the outer regions; an indication of this was already present in Bragaglia \& Tosi (2006) from the photometric metallicities, but it has recently been found on the basis of more precise metallicity determinations by Yong et al. (2005), Carraro et al. (2007), and Sestito et al. (2008).

Fig.1 (right panels) shows some results on the $[\alpha / \mathrm{Fe}]$ ratios found for clusters in our sample, based on our published OCs, on work in progress, on literature, and FLAMES data. The OCs follow the same relation of $[\alpha / \mathrm{Fe}]$ versus $[\mathrm{Fe} / \mathrm{H}]$ of the field thin disk stars and do not seem to show any trend with age. Perhaps the most interesting feature is 
shown in the bottom panel, where $[\alpha / \mathrm{Fe}]$ is plotted against $\mathbf{R}_{\mathbf{G C}}$ : from the clusters in the BOCCE sample there is no apparent trend of increased $[\alpha / \mathrm{Fe}]$ for the outer parts of the disk (see Sestito et al. 2008 for an extended discussion, since the two outermost clusters have abundances measured in that paper), at variance with what had been advocated by Carraro et al. (2004) and Yong et al. (2005), but in agreement with Carraro et al. (2007). However, we do not wish to make any strong statement until all clusters have been measured strictly on the BOCCE scale.

In summary, our effort to build a large, significant sample of open clusters with ages, distances, metallicities and detailed abundances measured with homogeneous methods is well under way and has already produced interesting results. Future efforts will be mainly directed towards increasing the number of clusters with chemical abundances measured on a common scale. Only the kind of homogeneity we are trying to achieve can guarantee that features are not created or lost because of systematic differences between analyses.

The work described here was made possible by the collaboration of many researchers, among them Gloria Andreuzzi and Luca Di Fabrizio (INAF - Fundación Galilei), Michele Cignoni (Bologna University), Jason Kalirai (UCO-Lick), and Gianni Marconi (ESO). We thank Paola Sestito and Sofia Randich for useful discussions; we make use here of results obtained in collaboration with them. We made large and fruitful use of the WEBDA (http://www.univie.ac.at/webda/). Generous allocation of observing time at Italian telescopes (TNG and Loiano), at the CFHT, and at ESO telescopes (La Silla and Paranal) is acknowledged.

\section{References}

Andreuzzi, G., Bragaglia, A., \& Tosi, M. 2008,A\&A, in press

Bragaglia, A., et al. 2001, AJ, 121, 327

Bragaglia, A. \& Tosi, M. 2006, AJ, 131, 1544

Bragaglia, A., Tosi, M., Carretta, E., Gratton, R. G., Marconi, G., \& Pompei, E. 2006a, MNRAS, 366,1493

Bragaglia, A., Tosi, M., Andreuzzi, G., \& Marconi, G. 2006b, MNRAS, 368, 1971

Bragaglia, A., Sestito, P., Villanova, S., Carretta, E., Randich, S., \& Tosi, M. 2008, A\&A, 480, 79

Bressan, A., Fagotto, F., Bertelli, G., \& Chiosi, C. 1993, A\& AS, 100, 647

Carraro, G., Bresolin, F., Villanova, S., Matteucci, F., Patat, F., Romaniello, M. 2004, ApJ, 128,1676

Carraro, G., Geisler, D., Villanova, S., Frinchaboy, P. M., \& Majewski, S. R. 2007, A\&A, 476, 217

Carretta, E., Bragaglia, A., Gratton, R. G., \& Tosi, M. 2004, A\&A, 422, 951

Carretta, E., Bragaglia, A., Gratton, R. G., \& Tosi, M. 2005, A\&A, 441, 131

Carretta, E., Bragaglia, A., \& Gratton, R. G. 2007, A\&A, 473, 129

D’Orazi, V., Bragaglia, A., Tosi, M., Di Fabrizio, L., \& Held, E. V. 2006, MNRAS, 368, 471

Dias, W. S., Alessi, B. S., Moitinho, A., \& Lépine, J. R. D. 2002, A\&A, 389, 871

Dominguez, I., Chieffi, A., Limongi, M., \& Straniero, O., ApJ, 524, 226

Friel, E. D. 1995, ARAA, 33, 381

Friel, E. D., Janes, K. A., Tavarez, M., Scott, J., Katsanis, R., Lotz, J., Hong, L., \& Miller, N. 2002, AJ, 124, 2693

Gratton, R., Bragaglia, A., Carretta, E., \& Tosi, M. 2006, ApJ, 642, 462

Randich, S., et al. 2005, ESO Messenger, 121, 18

Sestito, P., Bragaglia, A., Randich, S., Carretta, E., Prisinzano, L., \& Tosi, M. 2006, A\&A, 458, 121

Sestito, P., Bragaglia, A., Randich, S., Pallavicini, R., Andrievski, S. M., \& Korotin, S. A. 2008, $A \mathscr{E} A$, in press 
Tosi, M., Bragaglia, A., \& Cignoni, M. 2007, MNRAS, 378, 730

Twarog, B. A., Ashman, K. M., \& Anthony-Twarog, B. J. 1997, AJ, 114, 2556

Ventura, P., Zeppieri, A., Mazzitelli, I, \& D’Antona, F. 1998, A\&3A, 334, 953

Villanova, S., Carraro, G., Bresolin, F., Patat, F. 2005, ApJ, 130, 652

Yong, D., Carney, B. W., \& Teixera de Almeida, M. L. 2005, AJ, 130, 597

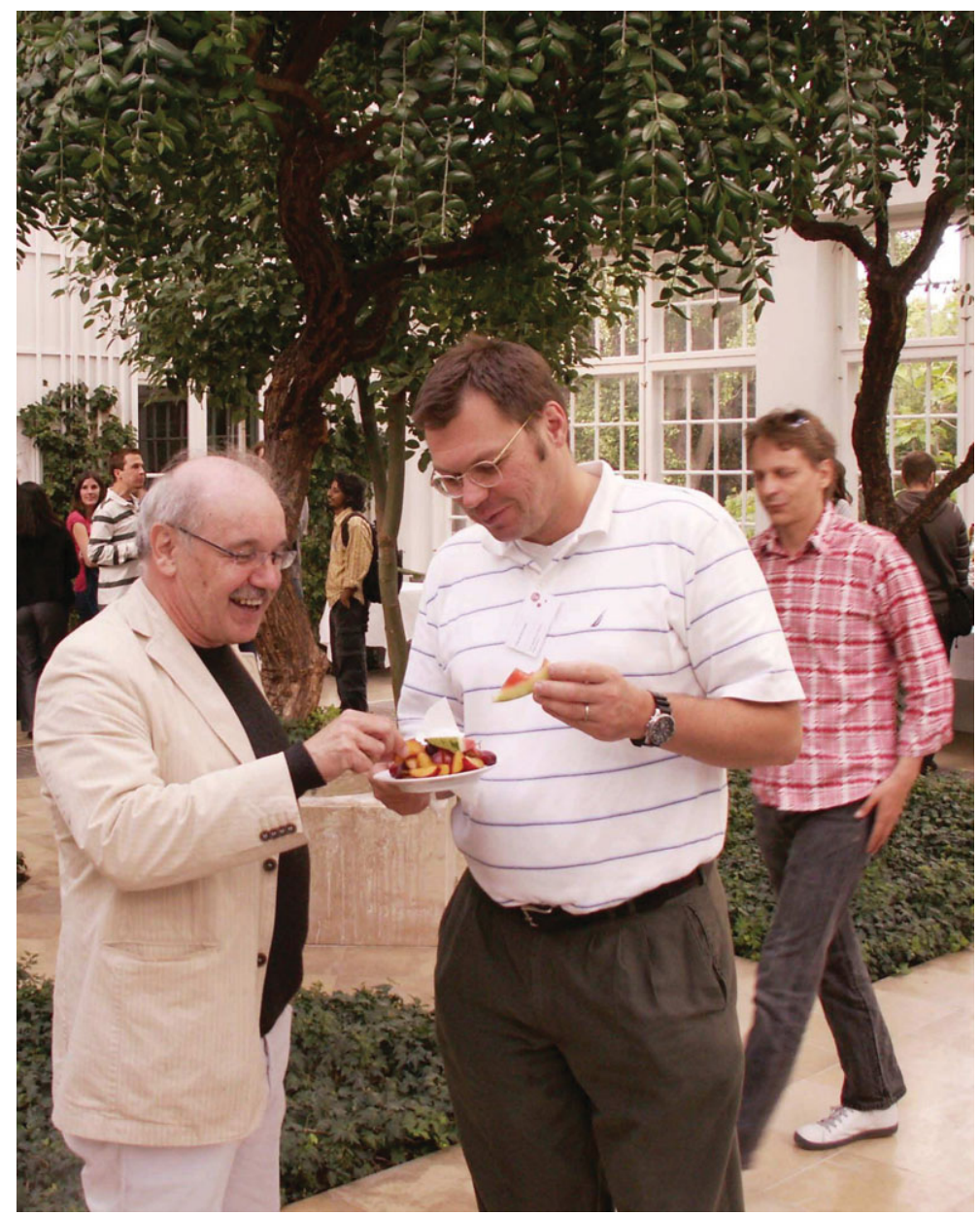

Hans Zinnecker and Pavel Kroupa in a joint accretion experiment at Carlsberg. Jens Hjorth in the background (photo: Bruce Elmegreen). 